\title{
Diagnóstico de síndrome de Cowden a partir de papilomatosis oral
}

Delgado W, Flores G, Concha H. Diagnóstico de sindrome de Cowden a partir de papilomatosis oral. Rev Estomatol Herediana 2006; 16(1): 46 - 52.

\section{RESUMEN}

El síndrome de Cowden o síndrome de hamartomas múltiples, es una rara genodermatosis de tipo autosómico dominante con expresividad variable. Los pacientes muestran una mezcla compleja de lesiones hamartomatosas de origen ectodérmico, mesodérmico y endodérmico, entre las que destacan pápulas en piel y mucosa oral, alteraciones en la tiroides, glándulas mamarias y tracto gastrointestinal. El diagnóstico temprano es de gran importancia, ya que los hombres afectados muestran alta susceptibilidad para desarrollar carcinoma de tiroides y las mujeres carcinoma de mamas. Este artículo describe las características de una paciente de 28 años de edad. cuyo diagnóstico de enfermedad de Cowden se estableció a partir del estudio clínico e histológico de pequeñas pápulas que presentaba en encías y dorso de la lengua. Asimismo, a la lista de alteraciones que componen el síndrome se agrega la presencia de siringomas faciales.

Palabras clave: SÍNDROME DE HAMARTOMA MÚLTIPLE / FIBROADENOMA / NEOPLASIAS DE LA TIROIDES / SIRINGOMA.

Diagnosis of Cowden's syndrome based on the identification of oral papillomatosis. ABSTRACT

Cowden's syndrome or multiple hamartoma syndrome is a rare dominant autosomic genodermatosis. Patients manifest a complex mixture of ectodermal, mesodermal, and endodermal hamartomatous lesions of which mucocutaneous, breast, thyroid and gastrointestinal tumors are most often encountered. Early diagnosis is of paramount importance since both affected men and woman show high susceptibilty to develop thyroid and breast carcinoma respectively. This article describe the clinical features of a female patient 28-year-old diagnosed of Cowden's syndrome based on the clinical and histologic study of small papulas found in the gums and in the dorsum of the tongue. Facial syringomas are added to the list of skin lesions that form the syndrome.

Keywords: MULTIPLE HAMARTOMA SYNDROME / FIBROADENOMA / THYROID NEOPLASMS / SYRINGOMA.
Wilson Delgado Azañero' Gabriel Flores Mena' Hiroshi Concha Cusihuallpa ${ }^{1}$

'Docente del Departamento Académico de Medicina, Cirugía y Patología Oral. Facultad de Estomatología. Universidad Peruana Cayetano Heredia.

\section{Correspondencia}

Wilson Delgado Azañero Av. Honorio Delgado 430 - Lima 31, Perú Teléfono: (511) 381-1950 e-mail:wdelgado@upch.edu.pe

Aceptado para publicación :

21 de setiembre del 2006.

\section{Introducción}

La enfermedad o Síndrome de Cowden (SC) o síndrome de hamartomas múltiples fue descrito por primera vez por Costello en 1940, en una mujer mexicana que murió de cáncer a la edad de 47 años (1). En 1963, Lloyd y Dennis (2) definieron y nombraron al síndrome, después de estudiar una paciente de 20 años de edad de nombre Raquel Cowden, quien presentaba facies adenoidea, arco palatino alto, hipoplasia del paladar blando y de la úvula, papilomatosis en los labios y orofaringe, lengua escrotal, lesiones en tiroides y enfermedad fibroquística de mamas, escoliosis y lesiones en los huesos e hígado, además a la edad de 16 años le habían diagnósticado enfermedad quística avanzada de mama con degeneración maligna. Por otro lado, en su familia se halló que una de sus hermanas había presentado un gran tumor en la tiroides, numerosos tumores pequeños en la piel de origen desconocido y padecido de retardo mental. También se encontró que dos de sus tías por parte de madre habían fallecido por carcinoma de mama.

Weary et al. (3) propusieron el nombre de síndrome de hamartomas múltiples, después de considerar que las le- siones tenían origen ectodérmico, mesodérmico y endodérmico. En 1977, Brownstein et al. (4) indicaron que los triquilemomas de la piel eran marcadores específicos de la enfermedad.

El SC es considerado una entidad muy rara, habiéndose estimado su prevalencia en 1/200,000 (5). Los diferentes estudios sobre esta enfermedad han establecido que se trata de una genodermatosis que se hereda como rasgo autosómico dominante con expresividad variable (712), cursa con hamartomas en la piel y mucosa oral, tracto gastrointestinal, huesos, sistema nervioso central, ojos y tracto génitourinario. El compromiso de la piel ocurre en el 90 a $100 \%$ de los casos (68,13,14), de glándula tiroides en el 88\%,(610) y durante el transcurso de la enfermedad se desarrollan neoplasias malignas, principalmente en tiroides y mamas. Algunos autores la incluyen dentro de las poliposis gastrointestinales hereditarias (10), con tendencia a degenerar en cáncer gastrointestinal (15).

Los estudios genéticos han determinado que un $80 \%$ de los pacientes son portadores de mutaciones en el gen supresor de tumores PTEN (phosphatase and tensin homolog deleted on chromosome 10) llamado también MMAC1 (mutated in multiple advanced cancers) o TEP1 (Transforming growth factor-regulated and epithelial cellenriched phosphatase) ubicado en el brazo del cromosoma 10q23. (16). El PTEN inhibe el crecimiento tumoral actuando como regulador del crecimiento celular potenciado por la tirosinkinasa (17), por otro lado, la proteína PTEN promueve la muerte celular, por lo tanto, su mutación produce sobreproducción de células dando lugar a los crecimientos hamartomatosos $(18,19)$. Una mutación idéntica del gen PTEN, se ha encontrado en el síndrome de Bannayan-Zonana, el cual comparte algunas características clínicas con la SC, aunque los pacientes tienen menor tendencia al cáncer, lo que sugiere que la mutación del gen PTEN no es el único factor responsable que conduce a la enfermedad $(20,21)$.

Si bien se admite que la enfermedad no tiene preferencia por género, Lee et al. (11) han señalado que es más frecuente en mujeres (60\%) y en la raza blanca (96\%), Las manifestaciones clínicas generalmente se inician en la segunda década de vida o a principios de la tercera (6), sin embargo; Tsubosa et al. (2), han demostrado en una serie de 9 casos japoneses que puede manifestarse desde el nacimiento hasta los 46 años, por otro lado, Lee et al. (11) y Willard et al. 
(22) han indicado que puede presentarse hasta los 75 años de edad. Es importante resaltar que el 50\% de pacientes tienen historia familiar de la enfermedad (15).

Las manifestaciones cutáneas corresponden a hamartomas representados por pápulas de 1 a 2 mm de tamaño que en la cara tienen una distribución periorificial (6-11, 23), histológicamente son diagnosticadas como triquilemomas; otra manifestación cutánea es la queratosis acral, constituida por pápulas queratósicas de 2 a $3 \mathrm{~mm}$ de tamaño ubicadas en la palma de la mano y planta de los pies. En la mucosa oral los hamartomas aparecen como múltiples pápulas de 1 a $2 \mathrm{~mm}$. de tamaño ubicadas principalmente en la encía y en la lengua, que histológicamente corresponden a hiperplasias fibroepiteliales (6, 7, 9-11, 13, 14). De acuerdo a la literatura las manifestaciones mucocutáneas son el motivo principal de consulta de los pacientes femeninos $(6,9,10)$.

A nivel de la cabeza se ha observado que el $80 \%$ de los pacientes presenta macrocefalia, 13\% alteraciones oculares como miopía y angiomas retinales $(6,8,13,24,25)$, los maxilares son pequeños, el paladar es ojival y existe pérdida prematura de dientes $(6,9,10,23)$. También se ha reportado mayor incidencia de caries $(9,23)$.

Las alteraciones de la glándula tiroides son muy importantes y se presentan en el 88\% de pacientes, manifestándose como bocio, adenomas, quiste del conducto tirogloso y adenocarcinoma folicular (6-9, 11). En mamas las lesiones más frecuentes son fibroquistes y fibroadenomas, llegando a presentarse en el 70-76\% de casos $(6,8)$. El 20-36\% de mujeres diagnosticadas con el síndrome desarrollan carcinoma de mama (6-9) y el 33\% lo hacen de forma bilateral (7). También se han reportado dos casos de cáncer de mama en varones (8).

En el tracto gastrointestinal el mayor número de alteraciones corresponde a poliposis intestinal. En el tracto genitourinario se han descrito teratomas, adenocarcinomas de la uretra y cuello uterino, carcinoma transicional, carcinoma de células renales y pólipos uretrales benignos, pero las lesiones más comunes son quistes ováricos y leiomiomas (14).

Entre las anormalidades esqueléticas se han diagnosticado quistes óseos, cifosis torácica, cifoescoliosis (6-9, 11, 13) y osteosarcoma (26). A nivel del sistema nervioso central se considera que la enfermedad de Lhermitte-Duclos, es parte del SC, la cual se manifiesta en la edad adulta y se caracteriza por la presencia de hamartomas en cerebelo, macrocefalia y ataxia cerebelar lentamente progresiva $(6,8)$.

La mortalidad en los pacientes con SC está relacionada con la frecuencia de cáncer que desarrollan, principalmente en mamas y tiroides, siendo mayor la incidencia de cáncer de mama en mujeres y de cáncer de tiroides en varones $(6,7)$. El pronóstico de estos pacientes es malo, ya que el $40-45 \%$ de ellos desarrollan al menos una neoplasia maligna $(6,8,10,13)$.

Debido a la amplia variedad de alteraciones que se pueden encontrar en el SC, la International Cowden Syndrome Consortium ha propuesto criterios operacionales para su diagnóstico (Tabla 1).

Para diagnosticar en una familia con SC a un individuo afectado (5), es suficiente encontrar:

1. Cuando existen solo lesiones mucocutáneas :

- Presencia de seis o más pápulas faciales de las cuales 3 o más son triquilemomas,

- Pápulas cutáneas faciales y papilomatosis oral,

- Papilomatosis oral y queratosis acral,

- Seis o más lesiones de queratosis palmar o plantar.

2. Dos criterios mayores siendo uno de ellos macrocefalia o enfermedad de Lhermitte-Duclos.

3. Un criterio mayor y tres menores

4. Cuatro criterios menores

El propósito de este trabajo es reportar el primer caso de SC descrito en el Perú, cuyo diagnóstico se estableció a partir del estudio clínico e histopatológico de las lesiones de la mucosa oral.

\section{Reporte del caso}

Paciente y métodos. Una paciente mujer de 28 años de edad, de raza mestiza con diagnóstico sistémico de enfermedad de Graves, fue referida al Servicio de Medicina y Patología Oral de la Facultad de Estomatología de la Universidad Peruana Cayetano Heredia, por presentar crecimiento "polipoides" en la lengua.

En el examen de la boca se encontraron múltiples pápulas de 1 a $2 \mathrm{~mm}$. de tamaño de superficie redondeada ubicadas en la cara vestibular y lingual de la encía marginal y adherida de ambos maxilares, partes laterales del paladar duro, en el límite del paladar duro y blando y en dorso y bordes laterales de la lengua (Fig. 1-4). También se encontraron en los ambos carrillos pápulas semejantes, distribuidas en forma dispersa, algunas más grandes, resultado de la confluencia de varias pequeñas La lengua se presentaba fisurada y se apreciaba una moderada protrusión bimaxilar.

En la piel de la frente, región suborbitaria y pómulos existían pequeñas pápulas de 1mm. de tamaño (Fig. 5, 6). En la palma de las manos y planta de los pies se detectaron pequeñas pápulas umbilicadas queratinizadas (Fig. 7).En el cuello, era evidente, a nivel de la tiroides, la presencia de un nódulo bien definido de 4 × 3 x 3 cms. de tamaño, de consistencia blanda, asintomático . Se decidió tomar biopsias de las lesiones de la mucosa oral y de la piel, cuyos diagnósticos histológicos fueron hiperplasia fibroepitelial y siringoma respectivamente (Fig. 8-11).

Tabla I. Criterios de diagnóstico operacionales propuestos por la International Cowden Syndrome Consortium. (ICSC)

\begin{tabular}{|c|c|c|}
\hline Criterios Patognomónicos: & Criterios mayores: & Criterios menores: \\
\hline Triquilemomas faciales & Carcinoma de mamas & $\begin{array}{l}\text { Lesiones de tiroides } \\
\text { (diferente a carcinoma) }\end{array}$ \\
\hline Queratosis acral & Carcinoma de tiroides (No medular) & Retardo mental ( $\leq$ a 75$)$ \\
\hline Crecimientos papilomatosos orales & Macrocefalia ( $\geq 97$ percentil) & Hamartomas gastrointestinales \\
\hline \multirow[t]{2}{*}{ Lesiones en diferentes mucosas } & $\begin{array}{l}\text { Enfermedad de } \\
\text { Lhermitte-Duclos }\end{array}$ & Enfermedad fibroquística \\
\hline & Carcinoma endometrial & $\begin{array}{l}\text { Lipomas, fibromas. Tumores o } \\
\text { malformaciones génitourinarias }\end{array}$ \\
\hline
\end{tabular}




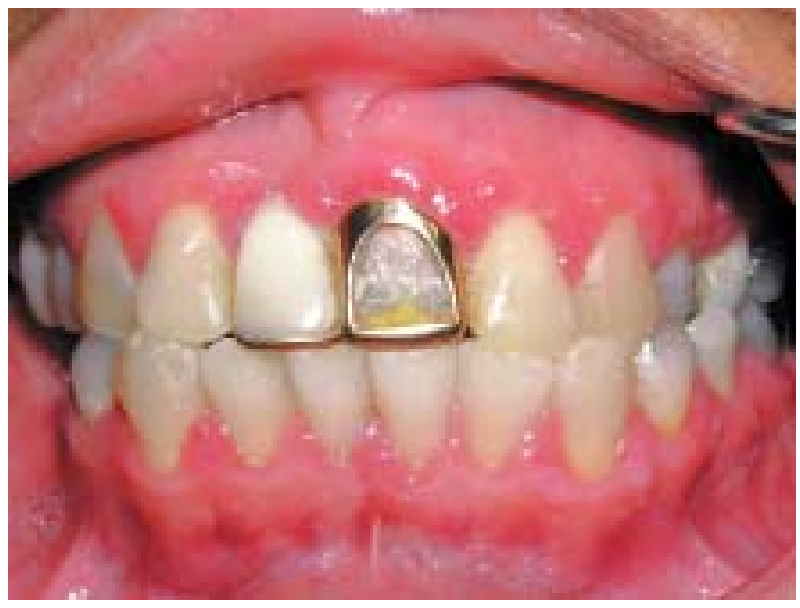

Fig. 1. Múltiples pápulas ubicadas en encía marginal y adherida.

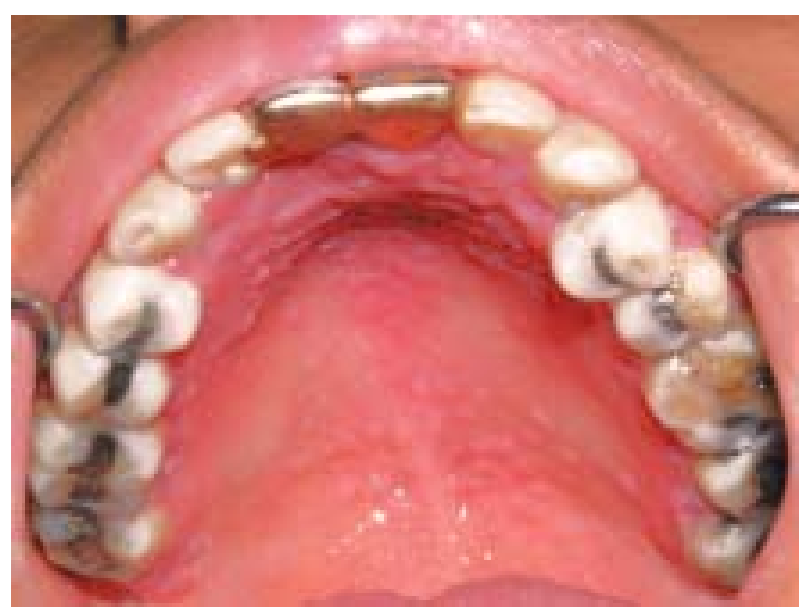

Fig. 3. Pápulas en el paladar distribuidas en forma de herradura.

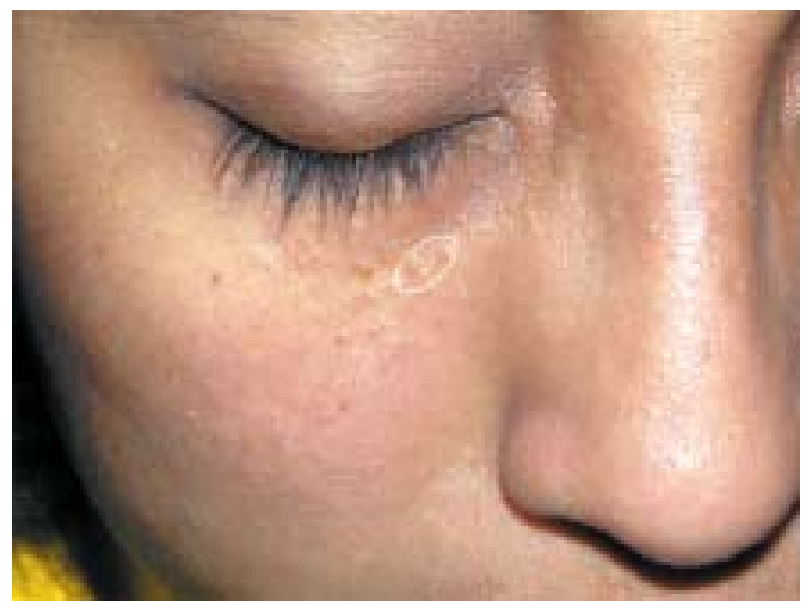

Fig. 5. Pequeñas elevaciones en piel de párpado inferior, que histológicamente corresponden a siringomas. (círculo)

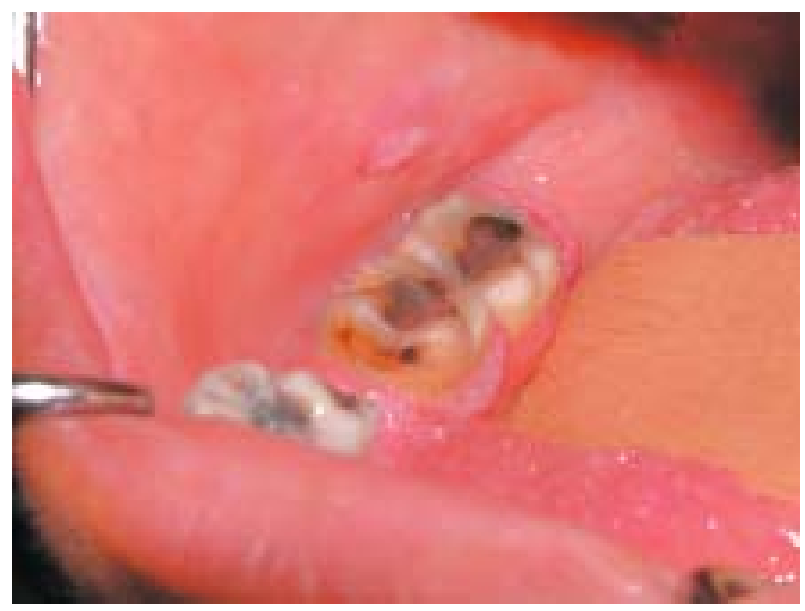

Fig. 2. Pápulas blanquecinas en encía adherida lingual y en carrillo.

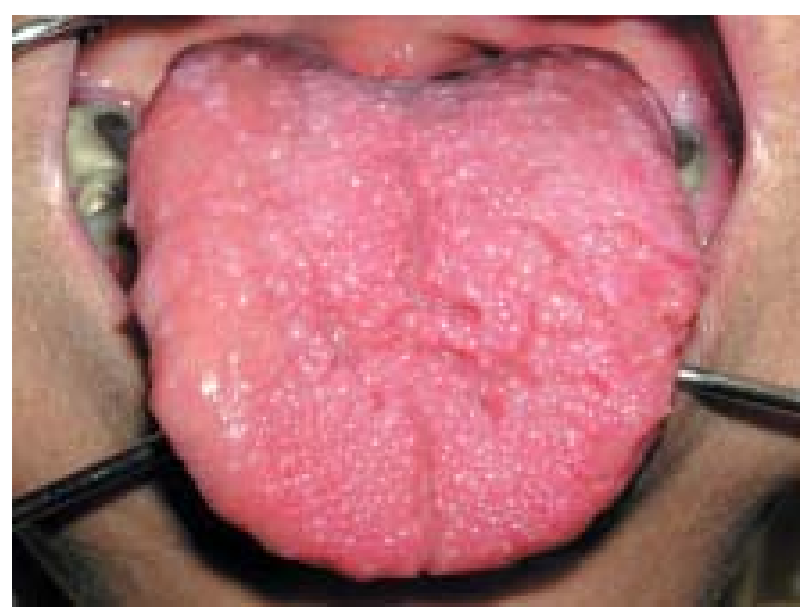

Fig. 4. Lengua fisurada y pápulas en el dorso y borde lateral de la lengua.

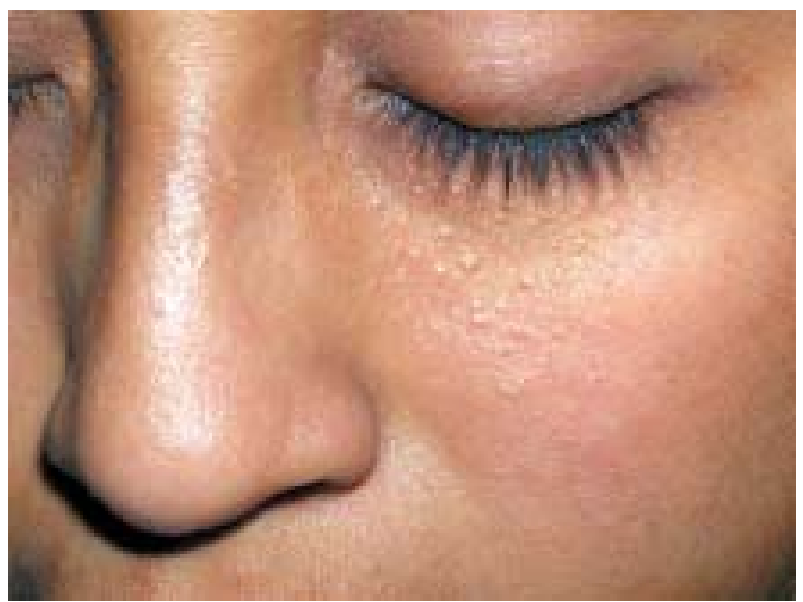

Fig. 6. Pápulas pequeñas de diferente tamaño en piel de párpado inferior, que corresponden a triquilemomas. 


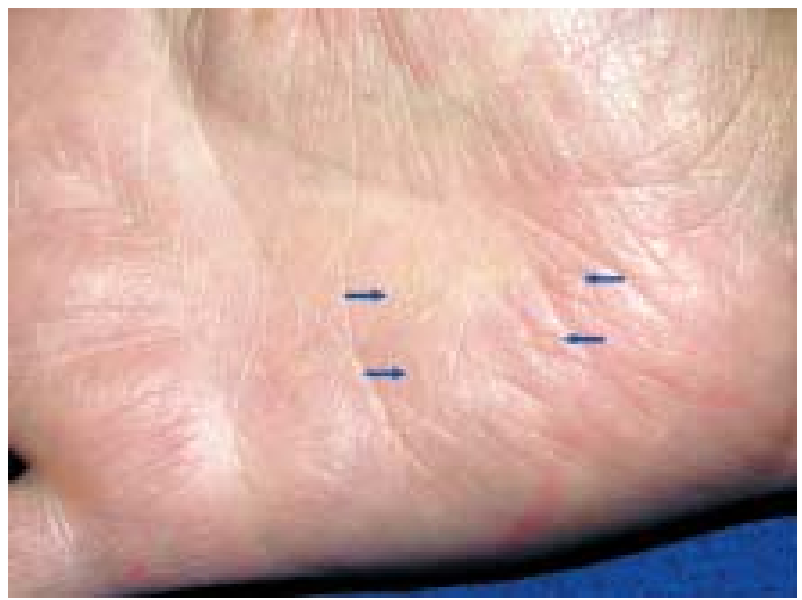

Fig. 7. Queratosis palmar (flechas).

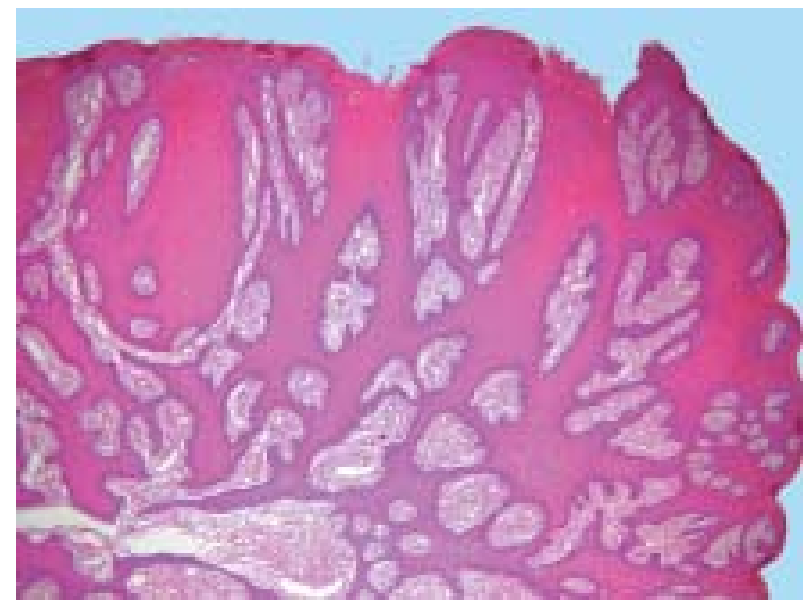

Fig. 8. Severa hiperplasia epitelial con clavos que se anastomosan formando una red. H\&E, x 40 (aumento original).

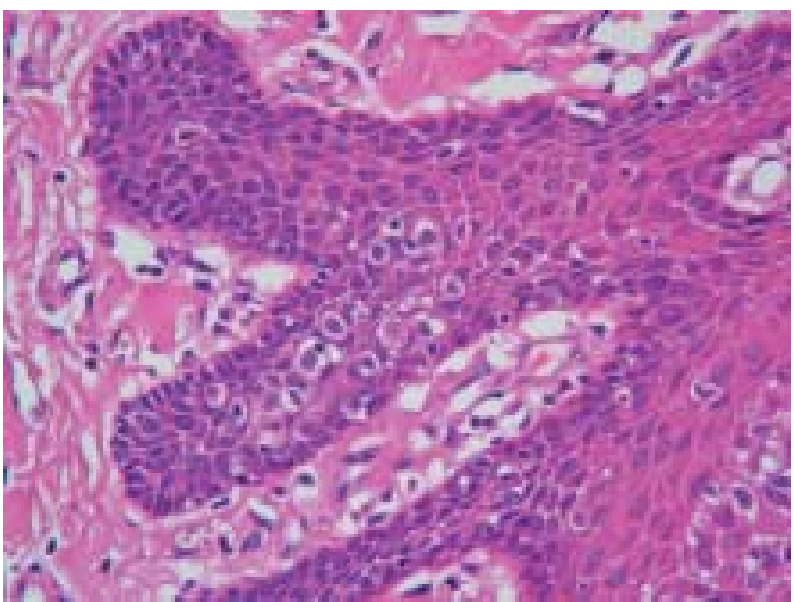

Fig. 10. Infiltrado de células mononucleares dentro del epitelio. H\&E, x 400 (aumento original).

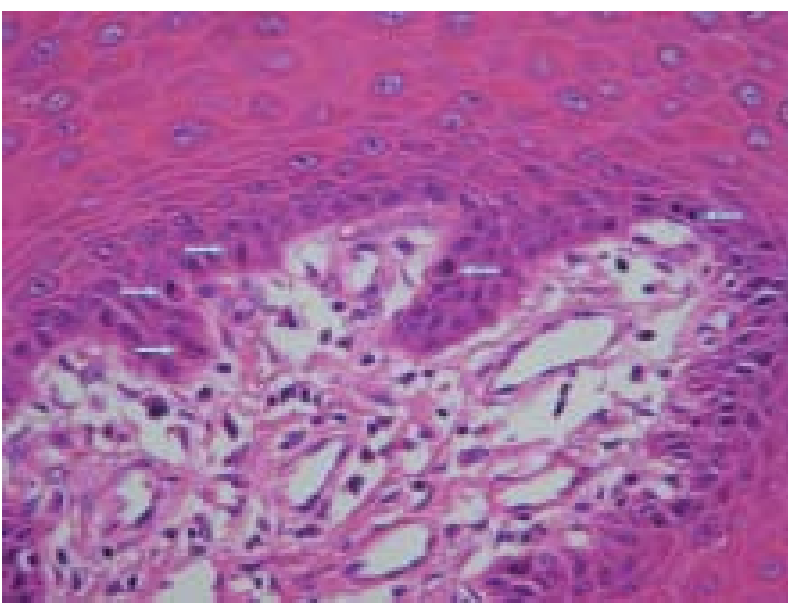

Fig. 9. Numerosas mitosis en la capa basal del epitelio (flechas). Múltiples capilares en el tejido conectivo. H\&E, x 400 (aumento original).

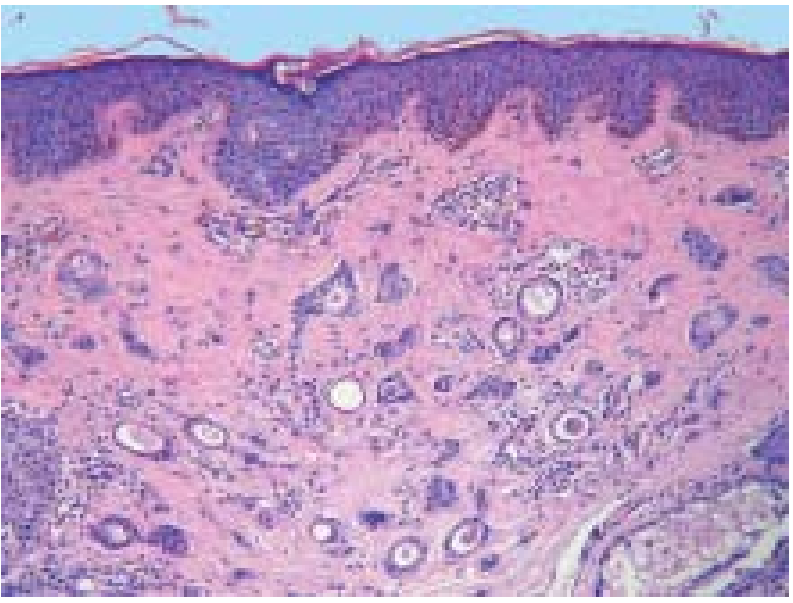

Fig. 11. Estructuras quísticas de conductos sudoríparos con material de secreción dentro de un estroma fibroso. H\&E, x 100 (aumento original). 
El análisis de los hallazgos clínicos e histológicos descritos condujo a la revisión completa de la historia clínica pasada de la paciente, obteniendo la siguiente información: a los 5 años había sido intervenida quirúrgicamente por un tumor benigno en la región costal derecha, cuyo diagnóstico patológico desconocía; a los 13 años le extirparon un tumor benigno en la mano derecha, cuyo diagnostico, según la paciente, fue quiste; a los 14 años le diagnosticaron tumores benignos en ambas mamas; a la edad de 26 años le extirparon un fibrolipoma de mama derecha y recibió tratamiento quirúrgico incompleto de un angioma venoso localizado en dorso de mano derecha. A los 27 años le detectaron un nódulo quístico en la tiroides cuyo diagnóstico anatomopatológico fue bocio coloide. Al momento de la consulta en nuestro Servicio la paciente tenía 28 años y estaba recibiendo tratamiento para hipertiroidismo con diagnóstico de enfermedad de Graves.

Sobre la base de los resultados de los estudios de las lesiones de la mucosa oral y de la piel de la cara, conjuntamente con los antecedentes patológicos descritos, se planteó el diagnóstico de Síndrome de Cowden. Se completó el estudio solicitándose reevaluación por las especialidades de ginecología, dermatología, gastroenterología y endocrinología. También se indicó estudio radiográfico del cráneo, hemograma, y dosaje de calcio, fósforo y fosfatasa alcalina.

En ginecología establecieron el diagnóstico de fibroadenoma de mama y papilomatosis intraductal; en dermatología se confirmó el diagnóstico de queratosis acral y triquilemomas periorbitarios; en endocrinología el estudio de la tiroides por gammagrafía estableció el diagnóstico de bocio difuso; en gastroenterología la endoscopía y colonoscopía se informaron sin alteraciones: los estudios hematológicos solicitados fueron normales; el estudio radiográfico del perfil del cráneo mostró protrusión bimaxilar.

Los hallazgos clínicos fueron analizados de acuerdo a los criterios propuestos por la International Cowden Syndrome Consortium (ICSC), estableciéndose el diagnóstico de Síndrome de
Cowden. La paciente fue informada acerca de las características evolutivas de su enfermedad y de la necesidad de mantener un control permanente sobre su estado de salud. Después de dos años de seguimiento, la paciente es vista en ginecología donde le detectaron un tumor en la mama derecha cuyo diagnóstico post operatorio fue carcinoma papilar infiltrante con márgenes de sección comprometidos, razón por la cual es sometida a tratamiento de quimioterapia. Actualmente la paciente se encuentra sin complicaciones.

\section{Discusión}

La revisión de la literatura aparecida en publicaciones indizadas en idioma inglés indica que hasta la fecha se han reportado un poco más de 200 casos de SC (6), incluyendo familias con varios miembros afectados (21). No hemos encontrado casos publicados en el Perú.

Es probable que, debido al desconocimiento que la presencia de hamartomas mucocutáneos múltiples y tumores benignos y malignos que se desarrollan en diversos órganos forman una unidad clínica, muchos casos de SC no sean diagnosticados oportunamente, más aun si se tiene en cuenta que por el curso benigno, muchas veces, las lesiones mucocutáneas, no son consultadas.

La característica más importante de los pacientes con síndrome de hamartomas múltiples es la alta posibilidad que tienen de cursar con patología neoplásica benigna y/o maligna en las glándulas tiroides y mamarias. La patología tiroidea es la mas frecuente habiéndose descrito en el $67 \%$ de los pacientes así, se han reportado casos con bocio coloide, adenoma folicular, oncocitoma, quistes del conducto tirogloso y en el $12 \%$ de los pacientes carcinoma de tiroides $(28,29)$. El caso que reportamos presentaba patología tiroidea benigna diagnosticada hace varios años como bocio coloide y recibía tratamiento para hipertiroidismo. La posibilidad que pudiera desarrollar en algún momento una neoplasia maligna de tiroides no se descarta.

Alrededor del 50\% de los pacientes de ambos sexos con SC desarrollan patología neoplásica benigna y maligna en las glándulas mamarias (30) dentro de los cuadros benignos se han descrito mastopatía fibroquística, fibroadenomas y malformaciones areolares y dentro de la patología maligna destaca el carcinoma de mama que puede aparecer hasta en un $36 \%$ de los pacientes $(1,5)$. La paciente de este reporte presentó fibroadenomas de mama y a los dos años de haberle diagnosticado SC desarrolló adenocarcinoma en la mama derecha.

Para prevenir la malignización de las lesiones de mamas en mujeres, se ha recomendado evitar el uso de estrógenos y realizar mamografías una vez al año, también se ha propuesto en algunos casos la mastectomía profiláctica $(10,13)$.

En el caso que se reporta el punto de partida para el diagnóstico de SC fue la identificación de pápulas orales múltiples distribuidas en varias zonas de la mucosa oral, diagnosticadas histológicamente como hiperplasias fibroepiteliales. La revisión de la historia médica pasada de la paciente así como los resultados de las interconsultas llevadas a cabo con diferentes especialidades médicas, permitieron identificar alteraciones en piel, glándula tiroides y mamas, las cuales analizadas bajos los criterios de la ICSC condujeron al diagnóstico de Síndrome de Cowden.

Las pápulas de la mucosa oral tenían uno a dos milímetros de tamaño, forma redondeada, color ligeramente más rosado que la mucosa normal, no producían ninguna molestia y estaban ubicadas en encías, dorso de la lengua, carrillos y paladar duro y blando, en estas últimas zonas se distribuían en forma de herradura (Fig. 3 ). Algunas pápulas de los bordes laterales de la lengua y del carrillo tenían mayor tamaño, probablemente como resultado de la fricción que estas zonas reciben durante la masticación. No se encontraron lesiones en borde libre del labio como las descritas por Capitan Cañadas et al. (32). En el diagnóstico clínico diferencial de las lesiones orales se consideró neuromas múltiples, enfermedad de Darier, acantosis nigricans hiperplasia epitelial focal y en último lugar linfangiomas capilares.

El estudio histológico de las pápulas orales correspondía a un tipo de creci- 
miento papilar muy particular, donde las proyecciones papilares corresponden a un epitelio hiperplásico paraqueratósi-co, cuya superficie es ligeramente redondeada. En el epitelio se distinguen zonas atróficas y zonas con marcada hiperplasia, esta última característica corresponde clavos epiteliales muy elongados anchos o delgados, los cuales se anastomosan en su parte terminal formando una red que encierra tejido conectivo con fibras colágenas delicadas y numerosos vasos sanguíneos de tamaño capilar o de pequeño calibre (Fig. 8)

Es importante destacar, que las células epiteliales presentaban un citoplasma marcadamente eosinófilo con puentes intercelulares muy definidos, además de mitosis en varias zonas de la capa basal y parabasal (Fig. 9 ), por otro lado, dentro del epitelio existían focos de un infiltrado mixto con predominio de células mononucleares.(Fig.10) Comparativamente, los cambios mas destacables ocurren en el tejido epitelial por lo que consideramos que las lesiones orales son epitelio-dependientes.

Las lesiones de mucosa oral no requieren tratamiento ya que son totalmente benignas y por que comprometen extensas zonas de la boca, sin embargo cuando están ubicadas el borde libre del labio, es recomendable extirparlas por razones estéticas.

$\mathrm{Al}$ igual que lo descrito en la literatura, en la boca se encontró lengua fisurada (3) (Fig. 4), pero no hubo evidencia de enfermedad periodontal ni de mayor tendencia a caries, aspectos que difieren de lo reportado en la literatura, donde se indica que existe mayor incidencia de caries; tampoco se halló paladar ojival (9). El estudio clínico y radiográfico del perfil del paciente estableció que presentaba protrusión bimaxilar moderada, que probablemente es también un componente del síndrome.

Las lesiones de la piel eran similares a las descritas en los casos reportados en la literatura, es decir presencia de queratosis palmoplantar y triquilemomas faciales ubicadas alrededor de las órbitas, adicionalmente es importante destacar que el estudio histológico de pequeñas pápulas faciales del lado derecho correspondió a siringomas (Fig.5 y 10 ). Este tipo de lesiones, diferentes histológicamente a los triquilemomas, pero en sus etapas iniciales clínicamente parecidos, no han sido descritas previamente en el SC, por consiguiente debe agregarse a la lista de lesiones cutáneas que constituyen el síndrome.

Para el tratamiento de las lesiones cutáneas se ha propuesto el uso del retinoide denominado acitretino, que es un análogo del ácido retinoico, cuyo mecanismo de acción parece estar relacionado con un incremento de la diferenciación celular (27). Cuando se considere usar esta droga, es importante tener en cuenta sus diversos aspectos negativos, como son: efecto teratógeno, producción de disfunción hepática, pancreatitis, xeroftalmia e inducción a la formación de pseudotumores cerebelares; por otro lado su eficacia es temporal y las lesiones pueden recurrir al interrumpirse el tratamiento (6).

Dentro de la amplia variedad a alteraciones que componen el SC destacan el desarrollo frecuente de cáncer de mama y de tiroides, neoplasias que pueden ser detectadas en etapas muy tempranas si se establece oportunamente el diagnóstico del síndrome. En este sentido el estomatólogo puede ser el primer profesional en identificar los fibropapilomas orales y los triquilemomas faciales, lesiones que se consideran patognomónicas del síndrome. Precisamente, el diagnóstico del presente reporte se estableció a partir del estudio y correcta interpretación de las lesiones orales.

Cuando se diagnostica SC, los pacientes y sus familiares deben ser informados acerca de las características evolutivas de la enfermedad y de la necesidad de ser examinados y controlados cada tres meses por las diferentes especialidades médicas, pero con particular énfasis por endocrinología, ginecología y gastroenterología. La supervivencia de los pacientes está directamente relacionada con el diagnóstico precoz de neoplasias malignas o de alteraciones complejas que pueden desarrollarse en diferentes partes del organismo.

Finalmente, deseamos señalar que el reporte que presentamos es un ejem- plo de la trascendencia que puede tener el diagnóstico e interpretación correcta de lesiones en la mucosa oral para la identificación de una enfermedad sistémica, en este caso de tipo hereditario, que cursa con neoplasias malignas.

\section{Referencias Bibliogáficas}

1. Salem OS, Steck WD. Cowden's disease (multiple hamartoma and neoplasia syndrome). A case report and review of the English literature. J Am Acad Dermatol 1983; 8(5):686-96.

2. Lloyd KM 2nd, Dennis M. Cowden's disease. A possible new symptom complex with multiple system involvement. Ann Intern Med 1963; 58(1):136-42.

3. Weary PE, Gorlin RJ, Gentry WC Jr, Comer JE, Greer KE. Multiple hamartoma syndrome (Cowden's disease). Arch Dermatol 1972; 106(5):682-90.

4. Brownstein $\mathrm{MH}$, Wolf M, Bikowski JB. Cowden's disease: a cutaneous marker of breast cancer. Cancer 1978; 41(6):2393-8.

5. Schreibman IR, Baker M, Amos C, McGarrity TJ. The hamartomatous polyposis syndromes: a clinical and molecular review. Am J Gastroenterol 2005; 100(2):476-90.

6. Miller C. Cowden disease (Multiple Hamartoma Syndrome) [Update 2005 June 2]. Disponible en: http:// www.emedicine.com/derm/ topic86.htm.

7. Tsubosa Y, Fukutomi T, Tsuda H, Kanai Y,Akashi-Tanaka S, Nanasawa T, Linuma G, Ushio K. Breast cancer in Cowden's disease: a case report with review of the literature. Jpn J Clin Oncol 1998; 28(1):42-6.

8. Maniglia JJ, Nicolau CCS, Catani GSA, Brotto ML, Maniglia R. Síndrome do hamartoma múltiplo (síndrome de Cowden): importância das manifestaçöes cutâneo mucosas para o otorrinolaringologista. Rev Bras Otorrinolaringol 1998; 64(6,pt.1):631-4.

9. Swart JG, Lekkas C, Allard RH. Oral manifestations in Cowden's syndrome. Report of four cases. Oral 
Surg Oral Med Oral Pathol 1985; 59(3):264-8.

10. Mignogna MD, Lo Muzio L, Ruocco V, Bucci E. Early diagnosis of multiple hamartoma and neoplasia syndrome (Cowden disease). The role of the dentist. Oral Surg Oral Med Oral Pathol Oral Radiol Endod 1995; 79(3):295-9.

11. Lee HR, Moon YS, Yeom CH, Kim KW, Chun JY, Kim HK, Choi HS, Kim DK, Chung TS. Cowden's disease-a report on the first case in Korea and literature review. J Korean Med Sci 1997; 12(6):570-5.

12. Gorlin RJ, Pindborg J, Cohen M Jr, editors. Syndromes of the head and neck. 2nd ed. New York: McGrawHill; 1976.

13. Takenoshita Y, Kubo S, Takeuchi T, Iida M. Oral and facial lesions in Cowden's disease: report of two cases and a review of the literature. $\mathrm{J}$ Oral Maxillofac Surg 1993; 51(6):6827.

14. Weinstock JV, Kawanishi H. Gastrointestinal polyposis with orocutaneous hamartomas (Cowden's disease). Gastroenterology 1978; 74(5 Pt 1):890-5.

15. Longy M, Lacombe D. Cowden disease. Report of a family and review. Ann Genet 1996; 39(1):35-42.

16. Marsh DJ, Coulon V, Lunetta KL, Rocca-Serra P, Dahia PL, Zheng Z, et al. Mutation spectrum and genotype-phenotype analyses in Cowden disease and BannayanZonana syndrome, two hamartoma syndromes with germline PTEN mutation. Hum Mol Genet 1998; 7(3):507-15.

17. McGarrity TJ, Wagner Baker MJ,
Ruggiero FM, Thiboutot DM, Hampel H, Zhou XP, Eng C. GI polyposis and glycogenic acanthosis of the esophagus associated with PTEN mutation positive Cowden syndrome in the absence of cutaneous manifestations. Am J Gastroenterol 2003; 98(6):1429-34.

18. Liaw D, Marsh DJ, Li J, Dahia PL, Wang SI, Zheng Z, et al. Germline mutations of the PTEN gene in Cowden disease, an inherited breast and thyroid cancer syndrome. Nat Genet 1997; 16(1):64-7.

19. Tsutsui $S$, Inoue $H$, Yasuda $K$, Suzuki K, Higashi H, Era S, Mori M. Reduced expression of PTEN protein and its prognostic implications in invasive ductal carcinoma of the breast. Oncology 2005; 68(46):398-404.

20. Wanner M, Celebi JT, Peacocke M. Identification of a PTEN mutation in a family with Cowden syndrome and Bannayan-Zonana syndrome. J Am Acad Dermatol 2001; 44(2):183-7.

21. Tok Celebi J, Chen FF, Zhang H, Ping XL, Tsou HC, Peacocke M. Identification of PTEN mutations in five families with Bannayan-Zonana syndrome. Exp Dermatol 1999; 8(2):134-9.

22. Williard W, Borgen P, Bol R, Tiwari $\mathrm{R}$, Osborne M. Cowden's disease. A case report with analyses at the molecular level. Cancer 1992; 69(12):2969-74.

23. Gertzman GB, Clark M, Gaston G. Multiple hamartoma and neoplasia syndrome (Cowden's syndrome). Oral Surg Oral Med Oral Pathol 1980; 49(4):314-6.
24. Gicquel JJ, Vabres P, Bonneau D, Mercie M, Handiri L, Dighiero P. Retinal angioma in a patient with Cowden disease. Am J Ophthalmol 2003; 135(3):400-2.

25. Porter S, Cawson R, Scully C, Eveson J. Multiple hamartoma syndrome presenting with oral lesions. Oral Surg Oral Med Oral Pathol Oral Radiol Endod 1996; 82(3):295-301.

26. Yen BC, Kahn H, Schiller AL, Klein MJ, Phelps RG, Lebwohl MG. Multiple hamartoma syndrome with osteosarcoma. Arch Pathol Lab Med 1993; 117(12):1252-4.

27. Cnudde F, Boulard F, Muller P, Chevallier J, Teron-Abou B. Cowden disease: treatment with acitretine. Ann Dermatol Venereol 1996; 123(11):739-41.

28. Eng C. Cowden syndrome. J Genet Couns 1997; 6(2):181-192.

29. Hemmings CT. Thyroid pathology in four patients with Cowden's disease. Pathology 2003; 35(4):311-4.

30. Starink TM. Cowden's disease: analysis of fourteen new cases. J Am Acad Dermatol 1984; 11(6):1127-41.

31. Walton BJ, Morain WD, Baughman RD, Jordan A, Crichlow RW. Cowden's disease: a further indication for prophylactic mastectomy. Surgery 1986; 99(1):82-6.

32. Capitan Canadas LM, Salinas Sanchez JL, Martinez Castillo SL, Labrot Moleon IL, Duran Moreno D, Sanchez Lopez D, Valencia Laseca E. Multiple oral fibropapillomatosis as an initial manifestation of Cowden Syndrome. Case report. Med Oral Patol Oral Cir Bucal 2006;11(4):E31924. 